\title{
Subgroups of chronic fatigue syndrome based on psychiatric disorder onset and current psychiatric status
}

\author{
Molly M. Brown, Carly Kaplan, Leonard A. Jason, Christopher B. Keys
}

Center for Community Research, DePaul University, Chicago, USA; mbrown59@depaul.edu

Received 22 October 2009; revised 2 December 2009; accepted 4 December 2009.

\begin{abstract}
Few studies have examined the effects of psychiatric disorders occurring over a long duration among patients with chronic fatigue syndrome (CFS). The role of premorbid and current psychiatric disorders in impairment was explored with a sample of 113 participants with CFS. Subgroups were created based on past and current psychiatric status including those whose psychiatric history was premorbid and current, postmorbid and current, past but not current, and those with no lifetime diagnosis. Results from a one-way MANOVA revealed that patients with a premorbid and current psychiatric disorder reported significantly higher pain severity, more somatic symptoms, poorer sleep quality, and poorer quality of life than those with no psychiatric history. Levels of fatigue and physical functioning among patients with CFS were unrelated to the four subgroups in this study. Although those with a premorbid and current psychiatric disorder were differentiated from those with no psychiatric history on some markers of impairment, the sample as a whole had severe fatigue-related impairment, which is the cardinal symptom of CFS. Implications for research are discussed.
\end{abstract}

Keywords: Chronic Fatigue Syndrome; Psychiatric Comorbidity; Subgroups; Impairment

\section{INTRODUCTION}

Chronic fatigue syndrome (CFS) is a chronic, debilitating illness that remains poorly understood. Some patients with CFS experience psychiatric symptoms, but the role of these symptoms in the development, maintenance, and severity of the illness is unclear. Several studies have found high rates of psychiatric comorbidity among patients with CFS in the range of $45 \%$ to $50 \%$, [1,
2] and up to $82 \%$ for a lifetime psychiatric diagnosis, [3] exceeding rates in the general population.

A number of studies have examined the role of comorbid psychiatric issues in the course of CFS. While one study found that patients with CFS who had a comorbid psychiatric diagnosis had significantly more severe fatigue than those without a psychiatric disorder, [4] most research has not found that psychiatric comorbidity increases impairment in CFS. Studies comparing patients with and without psychiatric comorbidity have found no differences in impairment in sleep, [5] neurocognitive functioning, $[6,8]$ physical functioning, $[9,11]$ fatigue severity, [5] and widespread pain. [11] These results suggest that psychiatric comorbidity is not related to higher illness severity in this population.

Due to the unexpected lack of evidence for a relationship between psychiatric comorbidity and illness severity in CFS, it is perhaps more important to explore long-term psychiatric status in CFS, as opposed to present psychiatric comorbidity only. Individuals with psychiatric illness early in life may be more likely to develop CFS later in life, [12] indicating that a subgroup of patients with CFS may have a long-term history of psychiatric disorder beginning prior to the onset of CFS. It has been hypothesized that an onset of psychiatric disorder predating CFS may be indicative of a long history of poor coping skills, leading to increased impairment. [13].

Tiersky, Matheis, DeLuca, Lange, and Natelson [13] developed psychiatric subgroups based upon whether the onset of the psychiatric disorder was before (premorbid) or after (postmorbid) CFS onset. They found that patients with CFS with a premorbid and current psychiatric diagnosis performed significantly worse on neuropsychological tests than patients with no history of psychiatric disorder and healthy controls. However, they did not find increased physical impairment in this subgroup. Tiersky et al. developed more specific psychiatric subgroups of patients with CFS compared to previous studies. Nonetheless, their study did not include patients who had a psychiatric disorder in the past but did not cur- 
rently meet criteria for the disorder. In other words, it may also be important to examine CFS severity for people who have recovered from a past psychiatric disorder, as remission from a psychiatric illness is associated with increased self-efficacy [14] and may be indicative of adaptive coping leading to better functioning. Further, the authors did not explore some key features of CFS severity such as sleep quality, pain severity, and diversity of symptoms.

The present study examined four groups of patients with CFS: those with a premorbid and current psychiatric diagnosis, those with a postmorbid and current psychiatric diagnosis, those with a past (either premorbid or postmorbid) but no current psychiatric diagnosis, and those with no history of psychiatric diagnosis. We hypothesized that patients with a premorbid and current psychiatric disorder would have increased physical and psychiatric impairment compared to patients with a postmorbid and current psychiatric diagnosis, those with a past but not current psychiatric disorder, and those with no history of psychiatric disorder.

\section{METHOD}

\subsection{Participants}

The present investigation used baseline data derived from a larger longitudinal study of non-pharmacological treatment interventions for CFS. [15] Participants were recruited from physician referrals, media advertisements, and CFS support groups.

Participants were at least 18 years of age, not pregnant, able to read and speak English, and considered to be physically capable of attending scheduled appointments. Participants were included if they met the Fukuda et al. [16] criteria for CFS (i.e., six or more months of persistent fatigue accompanied by four of the following eight symptoms: tender lymph nodes, sore throat, new or different headaches, muscle pain, joint pain, post-exertional malaise, unrefreshing sleep, and memory or concentration difficulties). Medical and psychiatric examinations were provided to rule out exclusionary medical or psychiatric diagnoses according to the Fukuda et al. criteria. In addition, participants in the current study must have provided self-reported date of onset for CFS and psychiatric diagnoses. A total of 113 participants were included in the present investigation. The DePaul University Institutional Review Board approved all procedures. All participants provided written informed consent.

\subsection{Materials}

\subsubsection{CFS Questionnaire}

The CFS Questionnaire was used to collect date of CFS onset, demographic, health status, medication usage, and symptom data. This screening scale has demonstrated adequate validity and inter-rater and test-retest reliability. $[17,18]$

Jason, Corradi, and Torres-Harding [19] explored 22 theoretically derived symptoms of CFS from the CFS Questionnaire for diagnostic importance. The authors found that these 22 symptoms are common among patients with CFS and are indicators of symptom clusters, including: neurocognitive (e.g., slowness of thought), vascular (e.g., dizzy after standing), inflammatory (e.g., allergies), muscle/joint (e.g., muscle pain), infectious (e.g., flu-like symptoms), and sleep/post-exertional (e.g., unrefreshing sleep) symptoms. This study utilized these 22 symptoms to measure total somatic complaints among participants. The total number of currently present symptoms endorsed by participants was used to determine the breadth of current symptoms experienced by participants.

\subsubsection{Structured Clinical Interview for DSM-IV (SCID)}

The SCID [20] was administered in order to establish Axis I psychiatric diagnoses. While a coding scheme is included in the instrument, the SCID allowed for clinical judgment in the assignment of symptoms to psychiatric or medical categories, a crucial distinction in the assessment of symptoms that overlap between CFS and psychiatric disorders, e.g., fatigue, concentration difficulty, and sleep disturbance. [21] A psychodiagnostic study [22] validated the use of the SCID in a sample of CFS patients. Through questioning about psychiatric symptom onset, an approximate date of psychiatric disorder onset is determined on the SCID.

\subsubsection{Medical Outcomes Study-Short Form-36 (SF-36)}

The SF-36, was used to measure self-reported functional status related to health. [23] The full measure included 36 self-report items that identified eight health concepts, or scales. This study used the Physical Functioning scale to measure overall disability. Higher scores indicated better health. Test construction studies for the SF-36 [24,25] found adequate internal consistency and discriminant validity across the eight scales of this measure.

\subsubsection{Fatigue Severity Scale (FSS)}

The FSS [26] was used to measure fatigue. This scale included 9 items rated on 7-point scales, and higher scores indicated more severe behavioral consequences of fatigue. Previous findings have demonstrated the utility of the FSS to discriminate between individuals with CFS, multiple sclerosis, and primary depression. [27] Within a CFS-like group, the FSS was found to be closely associated with severity ratings for the eight Fukuda et al. [16] CFS symptoms as well as with functional outcomes related to fatigue. [28] 


\subsubsection{Brief Pain Inventory (BPI)}

The BPI [29] was administered to measure the intensity of pain (pain severity) and the interference of pain in the patient's life (pain interference). This measure consisted of 14 questions with scores on each scale ranging from 0 to 10 . Higher scores indicated more pain. This measure exhibited adequate levels of reliability to assess pain in non-cancer samples, with alpha coefficients of .70 and above. [29] It also evidenced good concurrent validity with other generic pain measures. [30]

\subsubsection{Pittsburgh Sleep Quality Index (PSQI)}

The PSQI was a self-report measure developed to assess sleep quality in psychiatric research. [31] This index measured sleep disruptions and sleep quality. There were 19 questions (on 0-3 scale) which generated seven "component" scores: subjective sleep quality, sleep latency, sleep duration, habitual sleep efficiency, sleep disturbances, use of sleeping medication, and daytime dysfunction. The sum of scores for these seven components yielded one global score, which ranged from 0 to 21. Higher scores indicated worse sleep quality. The global scale demonstrated adequate internal consistency, test-retest reliability, and discriminant and concurrent validity. [31]

\subsubsection{Quality of Life Scale (QLS)}

The QLS measured satisfaction with different life activities for individuals with various chronic illnesses. [32] The scale consisted of 16 items answered on a Likerttype 1 to 7 scale asking how satisfied individuals are with each item. Higher scores indicated more overall life satisfaction. This scale demonstrated high test-retest reliability, convergent, discriminant, and construct validity among individuals with various stable chronic illnesses. [32]

\subsection{Procedure}

Once study eligibility criteria were met and no exclusionary illnesses were found based on the medical examination or SCID findings, participants completed self-report measures. The 113 participants were then divided into four psychiatric subgroups based on onset of psychiatric disorder in relation to CFS onset and current psychiatric status. In order to determine whether participants had a psychiatric disorder that predated the onset of CFS, the date of psychiatric disorder onset derived from the SCID was compared with self-reported date of CFS onset from the CFS Questionnaire. Specifically, participants with a current psychiatric disorder who reported having a psychiatric disorder at any point in their life prior to developing CFS were considered to have a premorbid and current psychiatric disorder $(\mathrm{n}=$ $32 ; 28.3 \%$ ). Those with a current psychiatric diagnosis and a history of psychiatric disorder that never predated CFS onset were considered to have a postmorbid and current disorder $(\mathrm{n}=12 ; 10.6 \%)$. Those with a history of psychiatric disorder who do not currently meet criteria for a psychiatric disorder comprised a third subgroup referred to past but not current psychiatric disorder $(\mathrm{n}=$ $28 ; 24.8 \%$ ). Finally, patients with no lifetime psychiatric diagnosis were placed into a fourth subgroup $(n=41$; $36.3 \%)$.

\subsection{Statistical Analyses}

Pearson chi-square analyses and one-way analysis of variance (ANOVA) were used to examine differences among the four psychiatric groups with regard to demographic variables. Fisher's Exact Tests were computed using SAS Proc Freq to compare the three groups with a history of psychiatric disorders on DSM-IV diagnostic categories. A one-way ANOVA was used to compare total number of lifetime psychiatric diagnoses between the three groups.

One-way multivariate analysis of variance (MANOVA) was used to test the primary hypotheses of this study with psychiatric group (premorbid and current, postmorbid and current, past but not current, and no lifetime psychiatric disorder) as the independent variable. The use of MANOVA reduced the likelihood of Type I error when making comparisons of multiple related dependent variables. [33] Upon examination of the correlation matrix of the outcome variables used in this study, all outcome variables were moderately correlated $(r \geq .3)$ with at least two other outcomes, indicating that MANOVA would be appropriate. Wilks' Lambda F approximation was used for interpretation of the multivariate test, and Bonferroni tests were used for post hoc comparisons.

\section{RESULTS}

\subsection{Demographic Characteristics}

In regards to demographic characteristics, $83.2 \%$ of participants were female. The average age was 43.8 years. Regarding ethnicity, $87.6 \%$ were White, $4.4 \%$ were African American, 4.4\% were Latino, and 3.5\% were Asian American. As for marital status, 48.7\% were married or living with a partner, $32.7 \%$ were single, and $17.7 \%$ were divorced or separated. In terms of work status, $40.7 \%$ were working or full time students and $59.3 \%$ were part-time students, retired, unemployed or on disability. With regards to education, $46.9 \%$ had earned a standard college degree, $22.1 \%$ had a graduate or professional degree, $21.2 \%$ had partial college, and $9.7 \%$ had a high school/GED degree or less. No significant 
Table 1. Lifetime Psychiatric Diagnoses for Psychiatric Subgroups.

\begin{tabular}{|c|c|c|c|c|}
\hline & $\begin{array}{l}\text { Premorbid and current } \\
(\mathrm{n}=32)\end{array}$ & $\begin{array}{l}\text { Postmorbid and current } \\
(\mathrm{n}=12)\end{array}$ & $\begin{array}{l}\text { Past but not current } \\
(\mathrm{n}=28)\end{array}$ & Sig. \\
\hline Any Mood Disorder & $59.4 \%$ & $58.3 \%$ & $50.0 \%$ & \\
\hline Major Depressive Disorder & $50.0 \%$ & $58.3 \%$ & $46.4 \%$ & \\
\hline Dysthymic Disorder & $18.8 \%$ & $0.0 \%$ & $0.0 \%$ & $*$ \\
\hline Other Mood Disorder & $0.0 \%$ & $0.0 \%$ & $3.6 \%$ & \\
\hline Any Anxiety Disorder & $56.3 \%$ & $33.3 \%$ & $46.4 \%$ & \\
\hline Generalized Anxiety Disorder & $9.4 \%$ & $8.3 \%$ & $3.6 \%$ & \\
\hline Panic Disorder (with or without Agoraphobia) & $25.0 \%$ & $16.7 \%$ & $17.9 \%$ & \\
\hline Posttraumatic Stress Disorder & $37.5 \%$ & $0.0 \%$ & $10.7 \%$ & $* *$ \\
\hline Other Anxiety Disorder & $6.3 \%$ & $8.3 \%$ & $10.7 \%$ & \\
\hline Adjustment Disorder & $21.9 \%$ & $33.3 \%$ & $7.1 \%$ & \\
\hline Other & $25.0 \%$ & $0.0 \%$ & $14.3 \%$ & \\
\hline Total Lifetime Psychiatric Disorders (Mean) & $1.97^{\mathrm{a}, \mathrm{b}}$ & $1.25^{\mathrm{a}}$ & $1.25^{\mathrm{b}}$ & $* * *$ \\
\hline
\end{tabular}

differences were found among the four groups in terms of demographic variables.

\subsection{Diagnostic Outcomes}

Total lifetime psychiatric diagnoses and rates of different psychiatric diagnostic categories were compared for the three groups with a history of psychiatric disorder (Table 1). Fisher's Exact Tests revealed that the three groups did not differ in terms of lifetime presence of anxiety or mood disorders. When comparing differences among the three psychiatric groups for specific psychiatric diagnoses, a significant difference was revealed for lifetime dysthymic disorder $(\mathrm{p}=.02)$, and the premorbid and current psychiatric group had the highest rate $(18.8 \%)$ compared to the other two groups with rates of $0 \%$. A significant difference between groups was also found for lifetime posttraumatic stress disorder $(p=.01)$. The premorbid and current group had the highest rate of posttraumatic stress disorder $(37.5 \%)$, the past but not current group had the second highest rate $(10.7 \%)$, and no participants from the postmorbid and current group met criteria during their lifetime.

A one-way ANOVA revealed that the groups significantly differed on total number of lifetime psychiatric diagnoses, $F(2,69)=9.19, p<.001$. Bonferroni post hoc analyses indicated that the premorbid and current psychiatric group had significantly more lifetime psychiatric diagnoses than both the postmorbid and current group ( $\mathrm{p}$ $=.01)$, and the past but not current group $(\mathrm{p}=.003)$.

\subsection{Main Outcomes}

Sixteen participants had missing data for one or more of the self-report outcome measures and were therefore excluded, which left a total of 97 participants for analysis of main outcomes (See Table 2). Results from the one-way MANOVA revealed a significant overall multivariate effect for psychiatric group on the combined DVs, Wilks' Lambda $=.68, \mathrm{p}=.03$.

Descriptive statistics for outcomes are reported in Table 2. Upon examination of univariate effects, significant differences were found for pain severity $[F(3,93)=$ $3.08, \mathrm{p}=.03]$, pain interference $[\mathrm{F}(3,93)=3.27, \mathrm{p}$ $=.03]$, total somatic symptoms $[\mathrm{F}(3,93)=3.86, \mathrm{p}=.01]$, sleep quality $[\mathrm{F}(3,93)=4.59, \mathrm{p}=.01]$, and quality of life $[F(3,93)=3.31, p=.02]$. No significant univariate effects were found for fatigue severity or physical functioning. Bonferroni post hoc tests revealed the premorbid and current group scored significantly worse than the no lifetime diagnosis group for pain severity $(\mathrm{p}=.02)$, pain interference $(p=.02)$, total somatic symptoms $(p=.03)$, sleep quality $(\mathrm{p}=.003)$, and quality of life $(\mathrm{p}=.02)$.

\section{DISCUSSIONS}

Patients with CFS with a premorbid and current psychiatric disorder reported significantly higher pain severity and interference, more somatic symptoms, poorer sleep quality, and poorer quality of life than those who have never been diagnosed with a psychiatric disorder. No significant differences in impairment were found for 
Table 2. Means (and Standard Deviations) for Psychiatric Subgroups on Outcome Measures.

\begin{tabular}{|c|c|c|c|c|c|}
\hline & $\begin{array}{l}\text { Premorbid and current } \\
(\mathrm{n}=29)\end{array}$ & $\begin{array}{l}\text { Postmorbid and current } \\
(\mathrm{n}=11)\end{array}$ & $\begin{array}{l}\text { Past but not current } \\
(\mathrm{n}=22)\end{array}$ & $\begin{array}{l}\text { No lifetime diagnosis } \\
(\mathrm{n}=35)\end{array}$ & Sig. \\
\hline Physical Functioning ${ }^{1}$ & $38.72(24.00)$ & $40.91(28.88)$ & $48.64(18.01)$ & $46.57(22.68)$ & \\
\hline Fatigue Severity ${ }^{2}$ & $6.19(1.01)$ & $6.29(0.64)$ & $6.16(0.71)$ & $5.98(0.69)$ & \\
\hline Pain Severity ${ }^{2}$ & $4.94(2.19)^{\mathrm{a}}$ & $3.98(2.39)$ & $4.30(1.58)$ & $3.33(2.31)^{\mathrm{a}}$ & $*$ \\
\hline Pain Interference ${ }^{2}$ & $5.33(3.15)^{\mathrm{a}}$ & $4.81(3.25)$ & $4.40(2.01)$ & $3.24(2.56)^{\mathrm{a}}$ & $*$ \\
\hline Somatic Symptoms ${ }^{2}$ & $16.21(3.56)^{\mathrm{a}}$ & $17.00(3.23)$ & $15.36(4.67)$ & $13.37(3.99)^{\mathrm{a}}$ & $* *$ \\
\hline Sleep Quality ${ }^{2}$ & $9.55(1.77)^{\mathrm{a}}$ & $8.36(3.29)$ & $8.05(2.28)$ & $7.49(2.23)^{\mathrm{a}}$ & $* *$ \\
\hline Quality of Life ${ }^{1}$ & $59.48(15.87)^{\mathrm{a}}$ & $69.91(18.11)$ & $65.68(12.42)$ & $71.03(15.16)^{\mathrm{a}}$ & $*$ \\
\hline
\end{tabular}

Notes: Similar letters across rows indicate significant difference; ${ }^{1}$ Higher numbers are better; ${ }^{2}$ Lower numbers are better; ${ }^{*} \mathrm{p}<.05, * * \mathrm{p}<.01$

participants in the other two psychiatric history groups compared to the no lifetime diagnosis group. These results in combination with two of Tiersky et al.'s [13] neuropsychological findings suggest that patients with CFS who have a premorbid and current psychiatric disorder have significantly more impairment than those with no psychiatric history, while patients with other categorizations of psychiatric history are not differentiated from patients without a psychiatric history in terms of impairment. However, the postmorbid and current psychiatric group did, in fact, demonstrate the highest level of fatigue severity and the most somatic symptoms compared to the other three groups, but significant differences were not revealed due to the small sample size for this group. Although patients with a premorbid and current psychiatric history were found to represent a subset of the CFS population that experiences particularly severe illness symptomatology, patients with a postmorbid and current diagnosis may also have increased illness severity.

Despite findings of increased impairment on some outcomes for those who had premorbid and current psychiatric diagnoses, no differences were found between groups for physical functioning or fatigue severity. Moreover, it is evident from this study that patients with and without psychiatric disorders exhibit notably high levels of fatigue and disability. These findings are consistent with previous research demonstrating that psychiatric comorbidity does not differentiate patients in terms of physical functioning [9] or fatigue severity. [5] Of note is that fatigue is the cardinal symptom of CFS, and findings from this and previous studies suggest that this symptom is present at a severe level regardless of psychiatric status.

In terms of severity of psychiatric functioning among patients with CFS, our prediction that the premorbid and current psychiatric group would evidence the most psychiatric dysfunction as defined by more lifetime psychiatric diagnoses was confirmed and was consistent with prior research. [13] This finding suggests that patients who have had ongoing mental health issues beginning prior to CFS onset tend to have more pervasive emotional problems. High levels of psychiatric impairment over time, in turn, may be related to increased illness-related impairment compared to those with a shorter-term history of psychiatric disorder.

The premorbid and current group had, on average, a total of 16.21 somatic symptoms compared to the no history group which had an average of 13.37 symptoms. This greater number of somatic symptoms suggests that the clinical presentation of patients with longstanding mental health issues is more complex than those without a psychiatric history. Exclusion of premorbid psychiatric disorder in CFS samples has been used to reduce sample heterogeneity in some studies. [34] Results from this study provide support for sample selection strategies that take into consideration premorbid psychiatric functioning, as long-term psychiatric status adds complexity to the illness symptomatology.

Several limitations can be noted for the present investigation. The use of self-reported onsets of psychiatric disorder and CFS to determine psychiatric subgroups may be problematic, as both are vulnerable to recall bias. $[35,36]$ Further, research has shown that those with a gradual CFS onset were more likely to report long-term depressive symptomatology gradually leading to the onset of the illness. [37] Thus, the self-reported onset of CFS in the present study may not have been accurate for all participants, particularly those who also had a gradual illness onset. Finally, differences between groups may not have been revealed in the analysis due to small sample sizes and low power. Future research with a larger sample size is needed to more fully explore functioning among patients with a postmorbid and current psychiatric diagnosis.

This study added to previous research demonstrating that neuropsychological functioning is impacted based on premorbid and current psychiatric status. [13] The 
findings indicated that long term psychiatric dysfunction increased impairment on several key indicators of CFS severity: sleep difficulties, pain, and wide ranging somatic symptoms. However, the sample as a whole had high levels of fatigue and physical impairment which were unrelated to the four subtypes, suggesting that even patients without a psychiatric history suffer from severe disability. Since the premorbid and current psychiatric history group also evidenced the most psychiatric dysfunction, treatment targeting mental health issues may help reduce symptom severity for this particular subgroup of patients. Past research suggests that examining current psychiatric status may not differentiate patient in terms of symptomatology. Based on the findings from this study, future research exploring the role of psychiatric functioning in CFS should examine psychiatric subgroups based on the onset and current status of the psychiatric disorder.

\section{REFERENCES}

[1] Johnson, S.K., DeLuca, J. and Natelson, B. (1996) Personality dimensions in the chronic fatigue syndrome: A comparison with multiple sclerosis and depression. Journal of Psychosomatic Research, 30, 9-0.

[2] Wessely, S., Chalder, T., Hirsch, S., Wallace, P. and Wright, D. (1996) Psychological symptoms, somatic symptoms, and psychiatric disorder in chronic fatigue and chronic fatigue syndrome: A prospective study in the primary care setting. American Journal of Psychiatry, 153, 1050-1059

[3] Buchwald, D., Pearlman, T., Kith, P., Katon, W. and Schmaling, K. (1997) Screening for psychiatric disorders in chronic fatigue and chronic fatigue syndrome. Journal of Psychosomatic Research, 42, 87-94.

[4] Jason, L.A., Taylor, R.R., Kennedy, C.L., Song, S., Johnson, D. and Torres, S. (2001) Chronic fatigue syndrome: Comorbidity with fibromyalgia and psychiatric illness. Medicine and Psychiatry, 4, 29-34.

[5] Morriss, R. K., Wearden, A. J. and Battersby, L. (1997). The relation of sleep difficulties to fatigue, mood and disability in chronic fatigue syndrome. Journal of Psychosomatic Research, 42, 597-605.

[6] Claypoole, K.H., Noonan, C., Mahurin, R.K., Goldberg, J., Erickson, T. and Buchwald, D. (2007) A twin study of cognitive function in chronic fatigue syndrome: The effects of sudden illness onset. Neuropsychology, 21, 507-513.

[7] Grafman, J., Schwartz, V., Dale, J.K., Scheffers, M., Houser, C. and Straus, S.E. (1993). Analysis of neuropsychological functioning in patients with chronic fatigue syndrome. Journal of Neurology, Neurosurgery, and Psychiatry, 56, 684-689.

[8] Joyce, J., Blumenthal, S. and Wessely, S. (1996) Memory, attention and executive function in chronic fatigue syndrome. Journal of Neurology, Neurosurgery, and Psychiatry, 60, 495-503.

[9] Ciccone, D.S., Busichio, K., Vickroy, M. and Natelson, B.H. (2003) Psychiatric morbidity in the chronic fatigue syndrome: Are patients with personality disorder more physically impaired? Journal of Psychosomatic Research, 54, 445-452

[10] Komaroff, A.L., Fagioli, L.R., Doolittle, T.H., Gandek, B., Gleit, M.A., Guerriero, R.T., et al. (1996) Health status in patients with chronic fatigue syndrome and in general population and disease comparison groups. The American Journal of Medicine, 101, 281-290.

[11] Morriss, R.K., Ahmed, M., Wearden, A.J., Mullis, R., Strickland, P., Appleby, L., et al. (1999) The role of depression in pain, psychological syndromes and medically unexplained symptoms associated with chronic fatigue syndrome. Journal of Affective Disorders, 55, 143-148.

[12] Harvey, S.B., Wadsworth, M., Wessely, S. and Hotopf, M. (2007) The relationship between prior psychiatric disorder and chronic fatigue: Evidence from a national birth cohort study. Psychological Medicine, doi: 10.1017/ S0033291707001900.

[13] Tiersky, L.A., Matheis, R.J., DeLuca, J., Lange, G. and Natelson, B.H. (2003) Functional status, neuropsychological functioning, and mood in chronic fatigue syndrome (CFS). Journal of Nervous and Mental Disease, 191, 324-331.

[14] Kavanagh, D.J. and Wilson, P.H. (1989) Prediction of outcome with group cognitive therapy for depression. Behaviour Research and Therapy, 27, 333-343.

[15] Jason, L.A., Torres-Harding, S., Friedberg, F., Corradi, K., Njoku, M.G., Donalek, J., et al. (2007). Non-pharmacologic interventions for CFS: A randomized trial. Journal of Clinical Psychology in Medical Settings, 14, 275-296.

[16] Fukuda, K., Straus, S.E., Hickie, I., Sharpe, M.C., Dobbins, J.G. and Komaroff, A. (1994) The Chronic Fatigue Syndrome: A comprehensive approach to its definition and study. Annals of Internal Medicine, 121, 953-959.

[17] Hawk, C., Jason, L.A. and Torres-Harding, S. (2007) Reliability of a chronic fatigue syndrome questionnaire. Journal of Chronic Fatigue Syndrome, 13, 41-66.

[18] Jason, L.A., Ropacki, J.A., Santoro, N.B., Richman, J.A., Heatherly, W., Taylor, R., et al. (1997). A screening scale for chronic fatigue syndrome: Reliability and validity. Journal of Chronic Fatigue Syndrome, 3, 39-59.

[19] Jason, L.A., Corradi, K. and Torres-Harding, S. (2007) Toward an empirical case definition of CFS. Journal of Social Service Research 34, 43-54.

[20] Spitzer, R.L., Williams, J.B.W., Gibbon, M. and First, M.B. (1995) Structured Clinical Interview for the DSM-IV-Non-Patient Edition (SCID-NP, Version 2.0). Washington, DC: American Psychiatric Press.

[21] Friedberg, F. and Jason, L.A. (1998) Understanding chronic fatigue syndrome: An empirical guide to assessment and treatment. Washington, D.C.: American Psychological Association.

[22] Taylor, R.R. and Jason, L.A. (1998) Comparing the DIS with the SCID: Chronic fatigue syndrome and psychiatric comorbidity. Psychology and Health, 13, 1087-1104.

[23] Ware, J.E. and Sherbourne, C.D. (1992) The MOS 36-item short-form health survey. Medical Care, 30, 473-483.

[24] McHorney, C.A., Ware, J.E. and Raczek, A.E. (1993). The MOS 36-Item Short-Form Health Survey (SF-36): II. Psychometric and clinical tests of validity in measuring 
physical and mental health constructs. Medical Care, 31, 247-263.

[25] McHorney, C.A., Ware, J.E., Lu, A.W. and Sherbourne, C.D. (1994) The MOS 36-item Short-Form Health Survey (SF-36): III. Tests of data quality, scaling assumptions, and reliability across diverse patient groups. Medical Care, 32, 40-66.

[26] Krupp, L.B., LaRocca, N.G., Muir-Nash, J. and Steinberg, A.D. (1989) The Fatigue Severity Scale: Application to patients with multiple sclerosis and systemic Lupus erythematosus. Archives of Neurology, 46, 1121-1123.

[27] Pepper, C.M., Krupp, L.B., Friedberg, F., Doscher, C. and Coyle, P.K. (1993) A comparison of neuropsychiatric characteristics in chronic fatigue syndrome, multiple sclerosis and major depression. The Journal of Neuropsychiatry and Clinical Neurosciences, 5, 200-205.

[28] Taylor, R.R., Jason, L.A. and Torres, S. (2000) Fatigue rating scales: an empirical comparison. Psychological Medicine, 30, 849-856.

[29] Cleeland, C.S. and Ryan, K.M. (1994) Pain assessment: The global use of the Brief Pain Inventory. Annals Academy of Medicine Singapore, 23, 129-138.

[30] Keller, S., Bann, C.M., Dodd, S.L., Schein, J., Mendoza, T.R. and Cleeland, C.S. (2004) Validity of the Brief Pain Inventory for use in documenting the outcomes of patients with noncancer pain. The Clinical Journal of Pain, 20, 309-18.

[31] Buysse, D.J. (1989). The Pittsburgh Sleep Quality Index:
A new instrument for psychiatric practice and research. Psychiatric Research, 28, 193-213.

[32] Burckhardt, C.S. and Anderson, K.L. (2003) The Quality of Life Scale (QOLS): Reliability, validity, and utilization. Health and Quality of Life Outcomes, 1, 60-63.

[33] Huberty, C.J. and Morris, J.D. (1989) Multivariate analysis versus multiple univariate analyses. Psycho-l ogical Bulletin, 105, 302-308.

[34] Natelson, B.H., Johnson, S.K., DeLuca, J., Sisto, S., Ellis, S.P., Hill, N., et al. (1995). Reducing heterogeneity in chronic fatigue syndrome: A comparison with depression and multiple sclerosis. Clinical Infectious Diseases, 21, 1204-1210.

[35] Kessler, R.C., Berglund, P., Demler, O., Jin, R. and Walters, E.E. (2005) Lifetime prevalence and age-of-onset distributions of DSM-IV disorders in the National Comorbidity Survey Replication. Archives of General Psychiatry, 62, 593-602.

[36] Bell, D.S. (1997). Illness onset characteristics in children with chronic fatigue syndrome and idiopathic chronic fatigue. Journal of Chronic Fatigue Syndrome, 3, 43-51.

[37] Salit, I.E. (1997). Precipitating factors for the chronic fatigue syndrome. Journal of Psychiatric Research, 31, 59-65. 